intensive support to fully establish. FSW engaged in self-help groups and supported each other by arranging child care, encouraging each other to go to get clinical care, establish savings and lending schemes and in some cases to return to educational or vocational training.

Conclusion Sex workers were empowered and able to make better life decisions. Priorities for the groups changed over time and as trust increased. Self-help-groups can become autonomous of programme support over time. Microplanning allowed us to regularly reach women not previously engaged in the programme. We plan to test the cost effectiveness of this intervention in a cluster randomised trial.

Disclosure No significant relationships.

\section{S05.4 \#SAVESEXY: A GAMIFIED APPROACH TO HARNESSING THE POWER OF COMMUNITY ACTIVISM FOR HIV TESTING PROMOTION}

Benedict Bernabe*. The Red Whistle, Makati, Philippines

10.1136/sextrans-2019-sti.35

As a response to the rising trend of new HIV infections among men who have sex with men (MSM), a group of volunteers in Manila, Philippines, formed a nonprofit organization called The Red Whistle (TRW). MSM in the Philippines suffer not just from HIV-related stigma but also from genderbased discrimination. In order to circumvent this environment, TRW devised '\#SaveSexy' a gamified approach to HIV awareness communication and HIV testing promotion that is not explicitly targeted to MSM but used visual imagery and messaging that would attract an MSM crowd. Using well-designed merchandise and branding, celebrity volunteers, and themed activities, '\#SaveSexy' encouraged its target audience to rethink the concept of 'sexy' as being confidently aware of their sexual health. The campaign takes its cue from market research done by TRW and partner ad agencies on what works for its target audience and applies this research to sexual health promotion. In this format, TRW partners with local government units and community-based organizations to organize 'races' where three teams of volunteers compete to encourage the most number of individuals to get tested in a single day. Elements of the strategy include teaming up with a celebrity volunteer and using social media to boost the reach and engagement of the information drive online. It also includes partnering with the local government's health office who will provide volunteers and materials for HIV testing. Aside from being well received, the intervention is also costeffective. Designed to cost at around Php 250,000 (USD $5,000)$ per event targeting 350 individuals tested, the average cost per is Php 715 (USD 14) per individual tested. In February 2019, it reached the most number of individuals tested in one day at 1,006, also for the lowest amount invested at Php 170,000 or USD 3,400 . Average cost per individual tested was Php 169 (USD 3.38).

Disclosure No significant relationships.
SO6 - IMPROVED MODELS AND TOOLS FOR STI INFECTIONS

\section{Monday, July 15, 2019 4:15 PM - 5:45 PM}

\section{S06.1 USE OF CERVICAL EXPLANTS TO STUDY GONOCOCCAL PATHOGENESIS}

Daniel Stein*. University of Maryland, Cell Biology and Molecular Genetics, College Park USA

\subsection{6/sextrans-2019-sti.36}

Gonococcal infections remain a challenging public health issue due, in part, to a lack of a vaccine. A major obstacle in vaccine development and for understanding gonococcal infections in women is the lack of tractable models mimicking in vivo infection in the female reproductive tract. We used human tissue-explants and isogenic gonococci (GC) to examine by quantitative imaging analysis the impact of the heterogeneity of cervical and bacterial surfaces on infection. We found that GC preferentially colonize the ectocervix and squamocolumnar junction (transformation-zone, TZ) but only penetrate into TZ and endocervical epithelia. Colonization of any region required the expression of pili. GC expressing Opacity-associated proteins (Opas) that bind the host carcinoembryonic antigen-related cell adhesion molecule (CEACAMs) (Opa $\left.a_{\text {CEA }}\right)$ increase ecto/endocervical colonization and reduce endocervical penetration. GC expressing Opas that bind heparan sulfate proteoglycans (HSPGs) (Opa $\mathrm{HSPG}_{\mathrm{HSP}}$ ) did not promote colonization or tissuer penetrationin any region of the cervix. Opa $a_{\mathrm{CEA}}$ inhibited GC-induced disruption of epithelial-epithelial adhesions and epithelial exfoliation, enhancing GC colonization and reducing penetration, through engaging CEACAMs. We propose the following model to explain GC pathogenesis of the female reproductive tract (FRT). GC establish colonization through pili-medieated adhesion. Opa ${ }_{\mathrm{CEA}}$ expression promotes colonization, leading to asymptomatic local infections. Low expression of $\mathrm{Opa}_{\mathrm{CEA}}$ allows GC to effectively penetrate into the endocervical epithelium, causing symptomatic infection.

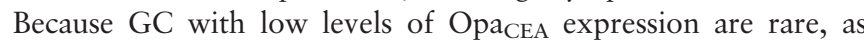

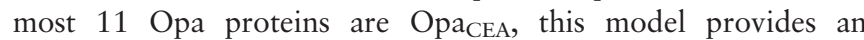
explanation as to why most infections of the FRT are asymptomatic and why invasive disease is rare.

Disclosure No significant relationships.

\section{S06.2 T. PALLIDUM IN VITRO GROWTH}

Steven I Norris*. McGovern Medical School at UTHealth, Pathology and Laboratory Medicine, Houston, USA

\subsection{6/sextrans-2019-sti.37}

For over a century, investigation of Treponema pallidum subsp. pallidum, the spiral-shaped bacterium that causes syphilis, was hindered by an inability to culture the organism in vitro. Recently, we reported long-term cultivation of this enigmatic 
organism using modifications of previously described mammalian cell co-culture. In vitro cultures of $T$. pallidum have now been maintained continuously for over 500 days, with full retention of multiplication rate, motility, structural integrity, and infectivity in a rabbit model. Genome sequencing of longterm in vitro cultured $T$. pallidum has revealed remarkable genetic stability, in that organisms from long-term in vitro culture had identical genome sequences and the same intrastrain heterogeneity observed in the original organisms used for inoculation. We have verified that replacement of Eagle's MEM with CMRL 1066 as the basal medium was key to achieving long-term growth. Surprisingly, the reducing agent dithiothreitol (DTT) was not required for long-term multiplication in the tissue culture system. We have also examined the effects of the scale of culture, medium composition, and axenic vs. mammalian cell co-culture. Finally, we have utilized limiting dilution to generate clonal isolates of T. pallidum, an important first step in developing a system to genetically manipulate the bacterium. Further development of the T. pallidum in vitro culture system is likely to have far-reaching effects on many aspects of $T$. pallidum research, including studies of physiology, structure, genetics, gene regulation, antimicrobial susceptibility, pathogenesis, immune reactivity, and epidemiology.

Disclosure No significant relationships.

\section{S06.3 PIGTAILED MACAQUE MODEL OF STIS}

Dorothy Patton*. University of Washington, Seattle, USA

\subsection{6/sextrans-2019-sti.38}

The Public Health Problem: Sexually transmitted infections (STIs) and their sequelae disproportionally affect young women, with cervical infections frequently ascending to the upper genital tract, leading to reproductive, pregnancy-related and newborn morbidity. Attributes of this Nonhuman Primate (NHP) as a Model: The pigtailed macaque (Macaca nemestrina) has several advantages over small animals for evaluating STIs, treatment and prevention. This nonhuman primate undergoes a regular menstrual cycle of 28-30 days and shows hormonal and genital tract changes similar to human females. Her microflora and reproductive tract tissues are similar in constituents and function to those of women. Use of the Model: The female pigtailed macaque model was initially developed in the early 1980's to simulate human Chlamydia trachomatis infection (cervicitis, salpingitis, pelvic inflammatory disease), pathogenesis and disease outcome, which has been key to our understanding of human chlamydial pathogenesis and treatment. The immune responses and histopathological characteristics of infection in this model closely resemble those seen in humans. This NHP model has been expanded to include lower genital tract infections with Trichomonas vaginalis, Mycoplasma genitalium and simian/human immunodeficiency virus (SHIV). Consequently, this model lends itself to co-infection studies using multiple STIs. Summary and Future Direction: M. nemestrina is naturally susceptible to multiple human sexually transmitted infections including C. trachomatis, T. vaginalis and M. genitalium. Pretreatment with exogenous hormones are not required to initiate or sustain these infections. Current model refinement efforts focus on modeling Neisseria gonorrhoeae infection. These STI pathogens are unique in that the majority of infections in women are asymptomatic, vaccines are currently unavailable, and concerns about antimicrobial resistance are on the rise. Supported by NIH and CONRAD HHSN2701700015I, N01 AI 95388, HHSN272201400016C, HHSN27220100006I, R21 AI 074898, P01 AI 39061, P51 OD010425 and MSA-02-315

Disclosure No significant relationships.

\section{S06.4 CHLAMYDIA, TRICHOMONAS AND SYPHILIS INFECTIONS IN MACAQUES: EFFECTS ON SIMIAN HIV ACQUISITION}

Ajay Sundaram Vishwanathan*. CDC (Centers for Disease Control and Prevention), Division of HIV and AIDS Prevention, Atlanta, USA

\subsection{6/sextrans-2019-sti.39}

Epidemiologic studies have linked sexually transmitted infections (STIs) to an increased risk of HIV acquisition. Although the precise mechanism of this association is unclear, it is likely to be a combination of STI-induced local inflammation, disruption of mucosal surfaces, and recruitment of HIV target cells. Given that some experiments are logistically difficult or impossible to conduct in humans, nonhuman primates (NHP) as STI models of enhanced HIV susceptibility are invaluable in understanding mechanisms, magnitude of risk, and evaluating effectiveness of biomedical interventions. Advantages of using NHPs over other animal models include their relatedness to humans and availability of better immunological reagents. We have successfully developed NHP models of both vaginal and rectal STIs, and studied them in the context of simian HIV (SHIV) acquisition and coinfection, and pre-exposure prophylaxis (PrEP) efficacy. We demonstrated that vaginal Chlamydia trachomatis (CT) and Trichomonas vaginalis (TV) infections increase SHIV acquisition risk while rectal CT infections do not. Also, to study efficacy of Truvada ${ }^{\circledR}$ (the only anti-HIV medication FDA-approved for PrEP), we used a validated STI-NHP model of repeated SHIV exposures to mimic populations at high risk for HIV infection, and demonstrated that oral Truvada ${ }^{\circledR}$ maintained efficacy despite CT-TV infections, albeit with a modest loss of PrEP activity. We showed that another promising anti-HIV injectable, long-acting cabotegravir, maintained complete efficacy against vaginal SHIV acquisition in NHPs infected with CT and TV. However, these are non-ulcerative infections, which led us to develop the first NHP models for rectally and vaginally acquired syphilis, an ulcerative STI. More NHP studies are ongoing to assess risk of vaginal SHIV acquisition and PrEP efficacy in macaques coinfected with syphilis, CT, and TV. These STI-NHP models are also powerful tools to study interactions between STIs, concomitant alterations in clinical manifestations and host responses, and to evaluate specific STIrelated interventions, including vaccines.

Disclosure No significant relationships. 